\title{
Intranasal Nerve Growth Factor administration improves cerebral functions in a child with severe traumatic brain injury: A case report
}

Antonio Chiaretti, Giorgio Conti, Benedetto Falsini, Danilo Buonsenso, Matteo Crasti, Luigi Manni, Marzia Soligo, Claudia Fantacci, Orazio Genovese, Maria Lucia Calcagni, Daniela Di Giuda, Maria Vittoria Mattoli, Fabrizio Cocciolillo, Pietro Ferrara, Antonio Ruggiero, Susanna Staccioli, Giovanna Stefania Colafati \& Riccardo Riccardi

To cite this article: Antonio Chiaretti, Giorgio Conti, Benedetto Falsini, Danilo Buonsenso, Matteo Crasti, Luigi Manni, Marzia Soligo, Claudia Fantacci, Orazio Genovese, Maria Lucia Calcagni, Daniela Di Giuda, Maria Vittoria Mattoli, Fabrizio Cocciolillo, Pietro Ferrara, Antonio Ruggiero, Susanna Staccioli, Giovanna Stefania Colafati \& Riccardo Riccardi (2017): Intranasal Nerve Growth Factor administration improves cerebral functions in a child with severe traumatic brain injury: A case report, Brain Injury, DOI: 10.1080/02699052.2017.1376760

To link to this article: http://dx.doi.org/10.1080/02699052.2017.1376760

曲 Published online: 03 Oct 2017.

Џ Article views: 8
Submit your article to this journal $₫$ \section{re}




\title{
Intranasal Nerve Growth Factor administration improves cerebral functions in a child with severe traumatic brain injury: A case report
}

\author{
Antonio Chiaretti ${ }^{a}$, Giorgio Conti ${ }^{b}$, Benedetto Falsini ${ }^{c}$, Danilo Buonsenso ${ }^{a}$, Matteo Crasti ${ }^{a}$, Luigi Manni ${ }^{d}$, Marzia Soligo ${ }^{d}$, \\ Claudia Fantaccia, Orazio Genovese ${ }^{\mathrm{b}}$, Maria Lucia Calcagnie, Daniela Di Giudae, Maria Vittoria Mattolie, \\ Fabrizio Cocciolilloe, Pietro Ferrara ${ }^{a}$, Antonio Ruggierof, Susanna Staccioli ${ }^{g}$, Giovanna Stefania Colafati ${ }^{\mathrm{h}}$, \\ and Riccardo Riccardif
}

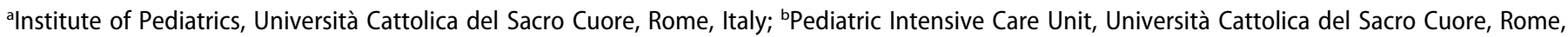

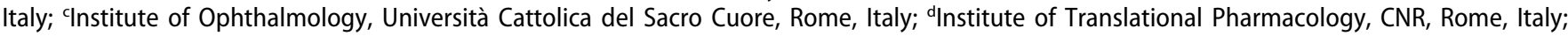

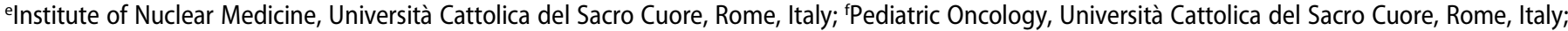
'Department of Neuroscience and Neurorehabilitation, Bambino Gesù Children's Hospital, Rome, Italy; ' Department of Imaging, Neuroradiology Unit, Bambino Gesù Children's Hospital, Rome, Italy

\begin{abstract}
Background: Nerve growth factor (NGF) promotes neural recovery after experimental traumatic brain injury (TBI) supporting neuronal growth, differentiation and survival of brain cells and up-regulating the neurogenesis-associated protein Doublecortin (DCX). Only a few studies reported NGF administration in paediatric patients with severe TBI.

Methods: A four-year-old boy in a persistent unresponsive wakefulness syndrome (UWS) was treated with intranasal murine NGF administration 6 months after severe TBI. The patient received four cycles of intranasal NGF $(0.1 \mathrm{mg} / \mathrm{kg}$, twice a day for 10 consecutive days).

Results: NGF administration improved functional [Positron Emission Tomography/Computed Tomography (PET/CT); Single photon emission/Computed Tomography (SPECT/CT) and Magnetic Resonance Imaging (MRI)] assessment, electrophysiological [Electroencephalogram (EEG) and Visual Evoked Potential (VEP)] studies and clinical conditions. He showed improvements in voluntary movements, facial mimicry, phonation, attention and verbal comprehension, ability to cry, cough reflex, oral motility, feeding capacity, and bowel and urinary functions. After NGF administration, raised levels of both NGF and DCX were found in the cerebrospinal fluid of the patient. No side effects were reported. Conclusions: Although further studies are needed for better understanding the neuroprotective role of this neurotrophin, intranasal NGF administration appears to be a promising and safe rescuing strategy treatment in children with neurological impairment after TBI.
\end{abstract}

\section{KEYWORDS}

Nerve Growth Factor; Doublecortin; traumatic brain injury; intranasal administration

\section{Abbreviations}

- BBB: Blood-Brain Barrier

- BDNF: Brain-Derived Neurotrophic Factor

- BFCN: Basal Forebrain Cholinergic Neurons

- CNS: Central Nervous System

- CSF: Cerebrospinal Fluid

- DCX: Doublecortin

- EEG: Electroencephalogram

- GCS: Glasgow Coma Scale

- HIBI: Hypoxic-Ischaemic Brain Injury

- MAD: Mucosal Atomiser Device

- NGF: Nerve Growth Factor

- MRI: Magnetic Resonance Imaging

- PET/CT: Positron Emission Tomography/Computed Tomography

- PICU: Paediatric Intensive Care Unit

- SPECT/CT: Single-Photon Emission/Computed Tomography
- SSEP: Somatosensory Evoked Potentials

- TBI: Traumatic Brain Injury

- TrkA: Tyrosine receptor Kinase A

- VEP: Visual Evoked Potential

\section{Introduction}

Traumatic brain injury (TBI) is a common cause of morbidity and mortality among children and young adults in developed countries. In 2010, the Centers for Disease Control and Prevention (CDC) estimated that TBI accounted for approximately 2.5 million emergency department (ED) visits and hospitalizations in the USA, either as an isolated injury or in combination with other injuries (1).

In particular, the rates of TBI-related ED visits increased for all age groups from the period of 2001-2002 to 2009-2010. The rates of TBI-related ED visits increased the most for children 4 years of age and younger. Children $0-4$ years of age had the

CONTACT Antonio Chiaretti achiaretti@yahoo.it E Institute of Pediatrics -Università Cattolica del Sacro Cuore, Rome, Italy.

Color versions of one or more of the figures in the article can be found online at www.tandfonline.com/ibij.

(4) Supplemental data for this article can be access on the publisher's website.

() 2017 Taylor \& Francis Group, LLC 
highest rates of any age group, typically with almost twice the rate of those in the next highest age group (15-24-year-olds) (2).

The outcome of TBI patients depends on the primary injury, for instance, the direct insult of neuronal, glial and vascular cells, and the secondary injury, which may include reperfusion, hypoxia, oedema and ischaemia (3). Secondary brain damage frequently causes the loss of cerebral neurons and can be associated with a progressive decrease in cognitive and motor functions. Children suffering from severe TBI show long-term sequelae, more pronounced in behavioural, neurological and neuropsychological functions (4). Currently, no effective therapies can restore neuronal loss or produce substantial clinical improvement in this kind of patients.

Nerve growth factor (NGF) is a neurotrophin supporting the neuronal growth, differentiation and survival (5) of brain cells and allows neurites to sprout, restoring the function of injured neurons (6). Experimental animal studies have shown that intraventricular NGF administration prevents the loss of phenotype and preserves cholinergic neurons after severe TBI, by increasing the synthesis of choline acetyltransferase and preventing atrophy of basal forebrain cholinergic neurons (7). Intraventricular NGF infusion has also been shown to reduce the malacic areas in the brain, improving cerebral perfusion and cognitive functions in children with severe hypoxicischaemic brain injury (4). However, little is known about the neuroprotective role of NGF in the damaged brain.

Some data indicate that NGF triggers the migration and proliferation of endothelial cells and enhances the functional maturation of newly assembled vessels and neuronal precursors $(8,9)$. Most of these neuronal precursors express morphological markers of striatum projection neurons, such as the Doublecortin (DCX) (10). DCX contributes to neuronal repair by stabilizing microtubules in neuronal cells and represents a specific marker for tracking the migration of new neurons into the injured sites of the brain (11). An increase of DCX expression has been demonstrated following intraventricular brain-derived neurotrophic factor (BDNF) and NGF administration (12). Despite the promising results obtained with NGF treatment, the poor permeability of the blood-brain barrier (BBB) when NGF is injected systemically (13) and the invasiveness of intraventricular administration represent the major obstacles to the development of NGFbased clinical trials. To the best of our knowledge, at least one clinical trial is registered and actually active in which adult patients with TBI should have been treated with intranasal NGF (14), but no results have been published yet. The intranasal delivery of NGF to the brain could represent a noninvasive and safe route to achieve a significant and effective concentration of NGF in selected brain areas (15). Given intranasally in rats with experimental TBI, NGF has been shown to reduce brain oedema and improve motor function $(16,17)$. Indeed, the olfactory pathway is a promising route for the direct delivery of NGF to the central nervous system (CNS), utilizing the potential transport pathway from the nose to the brain through the olfactory region with minimal systemic exposure $(18,19)$. According to these hypotheses, the aim of this case study is to investigate the effects of intranasal NGF administration on brain functions in a child with serious motor and cognitive impairment after severe TBI.

\section{Case Report}

A four-year-old boy, $20 \mathrm{~kg}$ body weight, was admitted to the Pediatric Intensive Care Unit (PICU) after a cardiac arrest following a severe TBI secondary to a car accident. He underwent immediate resuscitation manoeuvres and tracheal intubation. After 7 minutes of cardiopulmonary arrest, the patient returned to a spontaneous circulation and was transported to the PICU, where the neurologic examination showed a Glasgow Coma Scale (GCS) of 4. Total body CT scan showed blood share in the lateral ventricles, deep and diffuse haemorrhagic petechiae, occipital fracture, left pulmonary contusion and bilateral pleural effusion with flap. Brain MRI showed multiple frontal and temporal cortico-subcortical biemisferic haemorrhagic contusions, signs of axonal distraction at brainstem and at the splenium of the corpus callosum. Bilateral areas of increased T2 signal involving basal ganglia consistent with anoxic injury component were also detected. The spine MRI demonstrated severe oedematous spinal cord injury at the $\mathrm{C} 1-\mathrm{C} 2$ level. According to the post-resuscitation cardiac arrest management protocol established in our hospital, a 24hour mild therapeutic hypothermia $\left(34^{\circ} \mathrm{C}\right)$ was started. After 4 days the sedation was stopped and the child showed eye opening. A complete failure of respiratory trigger and a tetraplegia were detected, due to spinal cord and brain stem lesion, so the patient underwent tracheostomy and a placement of a gastrostomy tube. At day 28 after TBI the child showed a lack of swallowing, inability to speech, reflex movements without response to command and minimally conscious state in the presence of wakefulness [unresponsive wakefulness syndrome (UWS)] (20-22). Communication was possible only through eye movements and mouth opening. Somatosensory evoked potentials (SSEP) showed a marked distress functional tract of the upper limbs, presumably related to the damage at the brainstem and cervical spinal cord. Six months after TBI, the child was transferred to the department of Neuro-rehabilitation Unit for an overall rehabilitative management.

\section{Neurological and functional examination}

The neurological examination at the admission in Neurorehabilitation Unit showed an alert and conscious child with tetraplegia, severe communicative and neuropsychological impairment, and complete dysphagia. Spinal cord cervical injury resulted in impaired respiration requiring full-time mechanical ventilation through tracheostomy. The patient was fed only by gastrostomy. The child also lost bladder and bowel functions, due to the level of brainstem and spinal cord lesion. After an initial hypotonic state, increased muscle tone was observed, especially in the lower limbs. An oral-motor dyspraxia was also detected. Tetraplegia resulted in loss of function of the limbs, as well as the trunk, and pelvic organs. An accurate neurological assessment was difficult to obtain in view of the child's age, concomitant brain and spinal cord injuries, and severe disability in communication. Owing to these problems, a sensorimotor score was obtained using 'ASIA-IMSOP Classification', which revealed loss of skin sensitiveness and movement below the $\mathrm{C} 2$ level. The ASIA Impairment Scale of 'A' confirmed a complete spinal cord 
lesion (23). Only reflexed pattern movements were present. EEG recordings showed a theta background activity with a poor electric organization. Functional rating was performed using Spinal Cord Independence Measure (SCIM), Version III (24), reporting a final score of 11 (from 0 to 100, in which 100 represents the total independence in movements). A targeted management of spasticity with botulinum toxin injection in specific muscle groups was performed without any improvement. The patient also received numerous physiotherapy cycles without any evidence of improvement in his communicative and cognitive skills and or any of his motor functions.

\section{Intranasal NGF administration}

Six months after TBI, in view of the persistent severe UWS and tetraplegia and lacking any other feasible therapeutic approach (after proper and standardized medical, neuro-intensive and rehabilitative care), treatment with intranasal murine NGF administration was taken into consideration. This treatment was approved by our University's Ethical Board and by the child's parents, who provided written informed consent. The child received a total of four cycles of intranasal NGF. Each treatment was repeated at one-month intervals. In each cycle the NGF was administered at the dose of $0.1 \mathrm{mg} / \mathrm{kg}(2 \mathrm{mg}$ of NGF diluted in $20 \mathrm{~mL}$ of saline solution) through a mucosal atomiser device (MAD), used to deliver medication via a fine spray in both nostrils of the nasal cavity. In each treatment, $0.5 \mathrm{~mL}$ of the diluted solution was administered in each nostril ( $0.5 \mathrm{~mL}$ containing about $0.05 \mathrm{mg}$ of NGF twice a day for 10 consecutive days $=0.2 \mathrm{mg}$ of NGF/daily). This amount is considered sufficient to reach and stimulate NGF receptors, mainly Tyrosine receptor kinase A (TrkA), in most cerebral cholinergic and serotoninergic areas of the brain, as previously reported in the literature $(25,26)$. Before NGF administration, the nostrils were washed with $1 \mathrm{~mL}$ of saline solution, successively aspirated, to avoid any interference with the drug absorption (for the isolation and intranasal NGF preparation, see Supplementary Materials).

\section{Results \\ Clinical results}

After NGF treatment, significant improvements were observed in some cognitive processes, mainly in the planning of a communication strategy, attention and verbal comprehension. At the end of intranasal NGF administration, the patient showed a substantial improvement also in facial mimicry (enriched by new expressions such as joy, fear, wonder, etc.), with an initial capacity to knit eyebrows, curl nose, crying with tears (totally absent pretreatment), major symmetry in the smile and more meaning to eyelids closure in communicating. The child was also able to eat from the mouth; in fact, some improvements were also observed in oral motility and head rotation. At the admission in Neurorehabilitation Unit and after the fourth cycle of intranasal NGF, different functional motor scales were administered (T0-T4). CRSHC scale (Clinical Rating Scale for Head Control), House-Brackmann scale and Sunnybrook Facial
Grading Scale (Voluntary movement item) were tested and Range of head Movements (ROM) was observed. CRSHC at 0 and 4 Time remained grade 0 , the House-Brackmann scale remained unchanged at grade 2. Instead the Sunnybrook Facial Grading Scale (mouth opening) showed an improvement from score 2 (initiated slight movement) at T0 to score 4 (movements almost complete) at T4. The Range of Motion (head rotation) changed from left side Rom $9^{\circ}$ and right side Rom $3^{\circ}$ at $\mathrm{T} 0$ to left side Rom $12^{\circ}$ and right side Rom $6^{\circ}$ at T4. To detect the improvements in communicative function, a first language test (PING 'parole in gioco-test') was performed. The PING test is usually used to detect verbal comprehension using images in child with specific language disorders or cognitive disorder (includes a score of communicative skills from 19 to 37 months). At admission (T0), the child was not testable in relation to low attention level and the absence of a communicative tool, while after the fourth cycle of NGF treatment (T4) he arose a level of 37 months (maximum results). The child had a better relationship with his family members and all the caregivers and was less frustrated and in a better mood. Due to these neurological and clinical modifications, an alternative and increasing communication programme, involving all the family members, was started with great success. Oral motor dyspraxia progressively improved too, with enhanced oral motility control including mouth opening, tongue motility, mastication and swallowing. The ability to feed also improved and the child became able to eat little amounts of food with a parent-reported better discrimination regarding taste. In association with the improvement in oral motor dyspraxia, other acquired skills, included phonation with more explicit emission of sounds, were observed. Moreover, during the cycles of NGF therapy, a progressive but constant enhancement of head movements (mainly in head lateral rotation and minimally in up and down movements) was reported, while head support did not improve. Some hand finger movements during play with characteristics of voluntary control have been observed and even improved muscular tone and tropism. Another important amelioration was represented by the improvement in bowel function, loosing the need for stimulation to evacuate. The child also reacquired the cough reflex and hiccups, previously absent.

\section{PET/CT results (for protocol used, see Supplementary materials)}

The first PET/CT study was performed 6 months after head injury and 2 days before the beginning of intranasal NGF administration. The others PET/CT assessments were repeated the day after the end of each single cycle of intranasal NGF treatment and at one-month intervals. At visual analysis, the first PET/CT study showed a marked and global reduction of ${ }^{18}$ F-FDG uptake in all cortical and subcortical regions in both brain hemispheres, which was even more pronounced in the cerebellum, suggesting a severe and diffuse glucose hypometabolism (Figure 1, T0). After the first cycle of intranasal NGF administration, a significant increase of the ${ }^{18} \mathrm{~F}-\mathrm{FDG}$ uptake in the majority of brain regions was evident; only in both frontal superior regions the ${ }^{18}$ F-FDG uptake was only 


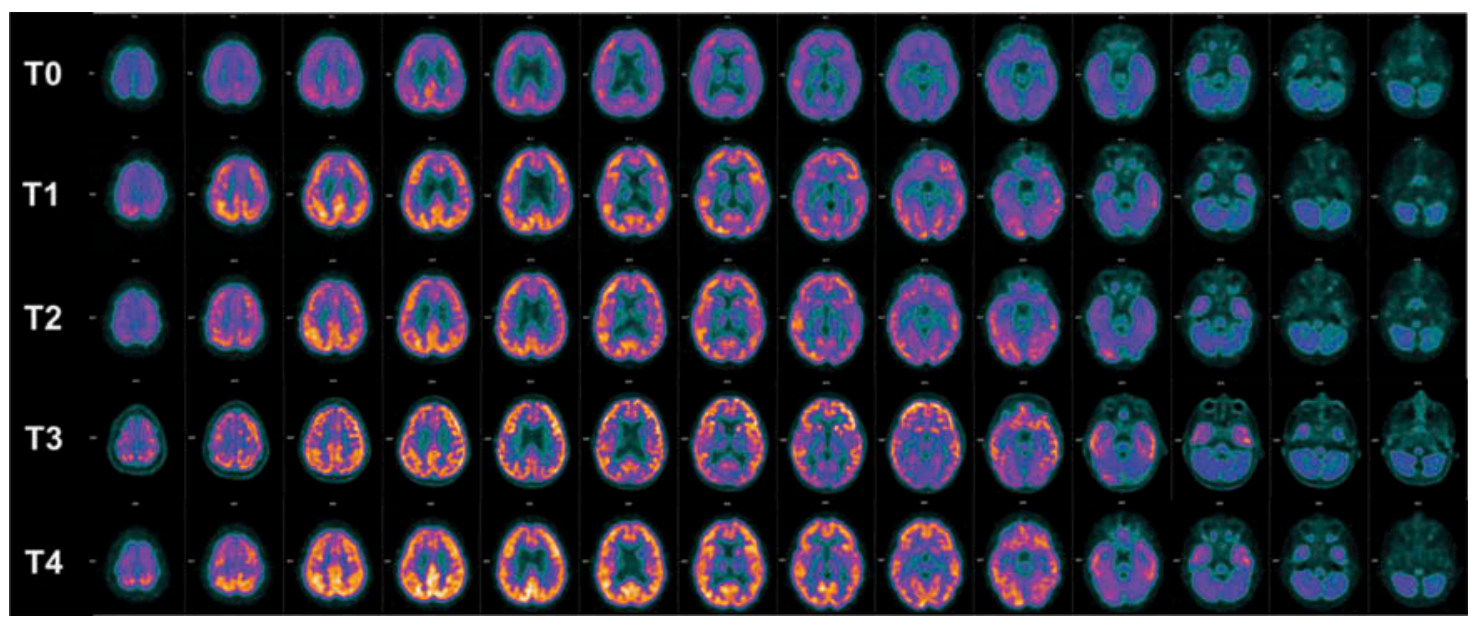

Figure 1. PET/CT study.

${ }^{18}$ F-FDG PET/CT images of brain transverse slices performed before NGF treatment (T0) and after the first (T1), the second (T2), the third (T3) and the fourth (T4) cycle of intranasal NGF administration. A severe global reduction of ${ }^{18} \mathrm{~F}$-FDG uptake in all cortical and subcortical regions in both brain hemispheres and, even more pronounced, in the cerebellum (T0) was observed. After the first NGF administration, a significant increase of the ${ }^{18} \mathrm{~F}$-FDG uptake in almost all subcortical and cortical regions was evident: only in both frontal superior regions the ${ }^{18} \mathrm{~F}$-FDG uptake was minimally increased and in the cerebellum it was almost unchanged (T1). After the second and the third cycle of intranasal NGF administration, the ${ }^{18} \mathrm{~F}$-FDG uptake in all subcortical and cortical regions remained substantially unchanged (T2, T3). After the last administration of intranasal NGF, a further increase of the ${ }^{18} \mathrm{~F}$-FDG uptake in all cortical and subcortical regions was evident, especially with the pretreatment PET/CT. A severe hypometabolism in the cerebellum and a moderate hypometabolism in the frontal superior regions still persist (T4). When comparing the last PET/CT (after the last intranasal NGF administration, T4) with the basal PET/CT (before starting the intranasal NGF administration, T0), an increase in glucose metabolic activity of at least $50 \%$ was found in all cerebral regions; only in the right and left superior frontal lobes and in the right and left cerebellum, the glucose metabolic activity increased by $41 \%, 41 \%, 30 \%$, and $32 \%$, respectively.

minimally increased and in the cerebellum it was almost unchanged (Figure 1, T1). This metabolic pattern persisted after the second and the third cycle (Figure 1, T2-T3). Finally, after the last intranasal administration of NGF, a further increase of the ${ }^{18} \mathrm{~F}-\mathrm{FDG}$ uptake was evident, especially when compared with the pretreatment PET/CT (Figure 1, T4). These findings suggested a progressive and gradual improvement of the brain glucose metabolism in all cortical and subcortical regions with the exception of both frontal superior regions and the cerebellum in which moderate and severe hypometabolism persisted, respectively. All these visual findings were confirmed by semi-quantitative analysis with maximum Standardized Uptake Value (SUV) calculated for each right and left cerebral Volumes of Interest (VOI), as reported in Table 1.

\section{SPECT/CT results (for protocol used, see Supplementary materials)}

The modalities and the timing of execution of SPET/CT assessments were the same as the PET/CT studies. Figure 2 shows the evolution of cerebral perfusion before and after the intranasal NGF administration. The baseline SPECT/CT scan (Figure 2, T0) revealed moderate hypoperfusion in the right frontal cortex and a mild hypoperfusion in the right anterior temporal and left frontal cortices, as well as in the cerebellar hemispheres. In the second SPECT/CT scan, performed after the first NGF treatment (Figure 2, T1), cerebral perfusion improved slightly in the right frontal and left frontal cortices, as well as in the right anterior temporal cortex, although it was more impaired in both cerebellar hemispheres. In the third and fourth SPECT/CT scans (Figure 2, T2 and T3), a further improvement was observed in the right and left frontal cortices and the right anterior temporal cortex. In the last SPECT/CT scan, at the end of NGF treatment (Figure 2, T4), the regional perfusion additionally improved in the right and left frontal cortices, the right anterior temporal cortex, the left occipital cortex and both cerebellar hemispheres. Visual interpretation of SPECT/CT images was confirmed by a semiquantitative analysis, using Statistical Parametric Mapping 8 (SPM8) for co-registration and spatial normalization purposes, and the mean radioactivity concentration was extracted by automatically defined VOI placed over the grey matter of both hemispheres and the cerebellum in normalized images. A statistical comparison by means of Fisher's exact test between VOI mean activity concentrations (expressed in percentages) obtained at T0 and T4 SPECT/CT scans showed a significant increase in radiotracer uptake in the right frontal

Table 1. Standardized Uptake Value (SUV) calculated for each right and left cerebral Volumes of Interest (VOI).

\begin{tabular}{|c|c|c|c|c|c|c|c|c|c|c|c|c|c|c|c|c|c|c|c|}
\hline \multirow[b]{2}{*}{ Time } & \multirow[b]{2}{*}{ SUV } & \multicolumn{2}{|c|}{ Temporal } & \multicolumn{2}{|c|}{ Parietal } & \multicolumn{2}{|c|}{ Frontal superior } & \multicolumn{2}{|c|}{ Frontal } & \multicolumn{2}{|c|}{ Occipital } & \multicolumn{2}{|c|}{ Cerebellum } & \multicolumn{2}{|c|}{ Caudate } & \multicolumn{2}{|c|}{ Putamen } & \multicolumn{2}{|c|}{ Thalamus } \\
\hline & & Right & Left & Right & Left & Right & Left & Right & Left & Right & Left & Right & Left & Right & Left & Right & Left & Right & Left \\
\hline T0 & SUVmax & 4.53 & 4.39 & 2.46 & 2.16 & 1.99 & 2.03 & 2.76 & 2.87 & 4.28 & 3.94 & 2.89 & 2.75 & 3.67 & 4.14 & 3.01 & 3.15 & 2.58 & 2.41 \\
\hline T1 & SUVmax & 5.21 & 5.58 & 5.75 & 5.33 & 2.75 & 2.74 & 3.97 & 3.98 & 6.26 & 5.42 & 3.26 & 2.98 & 4.18 & 5.37 & 3.89 & 3.52 & 3.89 & 3.81 \\
\hline $\mathrm{T} 2$ & SUVmax & 7.12 & 7.73 & 6.47 & 7.3 & 2.81 & 2.89 & 5.86 & 5.83 & 6.29 & 6.77 & 3.67 & 3.57 & 5.82 & 6.02 & 4.47 & 4.35 & 4.37 & 4.65 \\
\hline T3 & SUVmax & 7.82 & 7.93 & 7.21 & 7.75 & 2.8 & 2.85 & 6.34 & 6.35 & 6.37 & 6.09 & 3.61 & 3.52 & 6.24 & 6.7 & 4.68 & 4.42 & 4.95 & 4.91 \\
\hline $\mathrm{T} 4$ & SUVmax & 7.54 & 7.07 & 7.79 & 8.03 & 2.81 & 2.88 & 6.16 & 6.69 & 7.4 & 7.89 & 3.76 & 3.65 & 6.52 & 6.66 & 4.73 & 4.75 & 5.14 & 5.45 \\
\hline
\end{tabular}




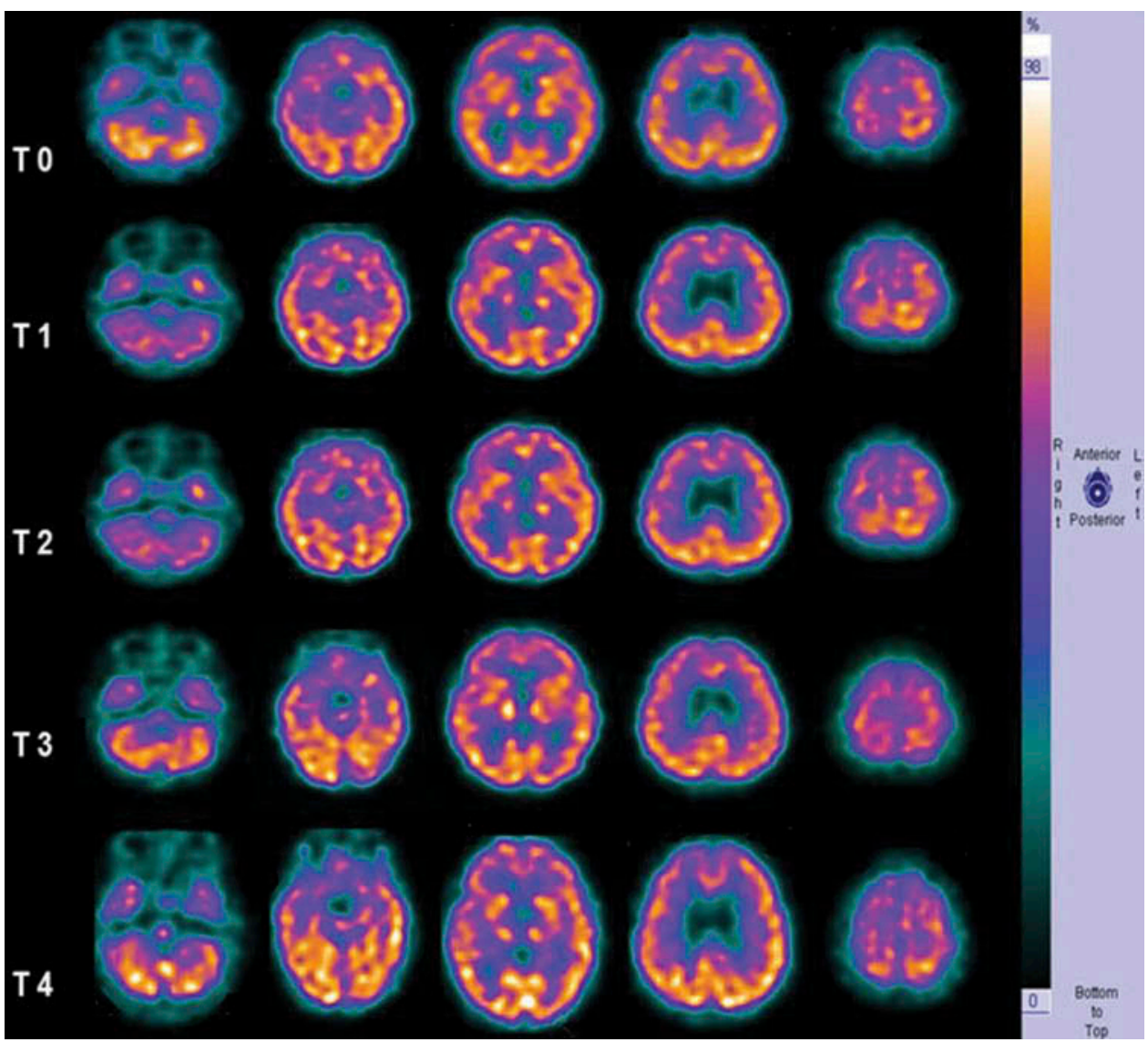

Figure 2. SPECT/CT study.

99mTc-HMPAO SPECT/CT images before (T0) and after intranasal NGF administration (T1, T2, T3and T4). An increase in the radiotracer uptake was found in the right frontal $(+19 \%, p=0.01)$, right anterior temporal $(+35 \%, p=0.007)$, left frontal $(+8 \%, p=0.30)$ and left occipital $(+13 \%, p=0.01)$ cortices, as well as in the right and left cerebellar hemispheres $(+18 \%, p=0.03$ and $+12 \%, p=0.07$, respectively), by calculating the percentage differences in the mean activity concentration of the selected VOIs between T0 and T4 SPECT/CT scans.

cortex $(p=0.01)$, the right anterior temporal cortex $(p=0.007)$, the left occipital cortex $(p=0.01)$ and the right cerebellar hemisphere $(p=0.03)$. Differences in VOI mean activity concentrations between $\mathrm{T} 0$ and $\mathrm{T} 4$ measurements failed to reach statistical significance in the left frontal cortex $(p=0.30)$ and the left cerebellar hemisphere $(p=0.07)$.

\section{BRAIN AND SPINAL MRI (for protocol used, see Supplementary materials)}

Figure 3 shows brain and spinal MRI assessed 6 months after TBI. Brain parenchymal modifications before and after NGF intranasal administration were reported. The first MRI was performed 5 days before the beginning of intranasal NGF treatment. The last MRI was carried out 1 week after the end of intranasal NGF therapy. The first brain MRI showed multiple focal traumatic lesions and haemorrhagic contusions in the frontal and temporal cortico-subcortical regions, signs of axonal distraction at the brainstem and at the splenium of the corpus callosum, as consequences of TBI. Areas of increased T2 signal and decreased T1 signal involving the basal ganglia consistent with anoxic injury component were also detected (Figure 3: a-c). The MRI assessment after NGF treatment showed the evolution of the size of the ventricles and the subarachnoid spaces, demonstrating a significant reduction in both ventriculomegaly and subarachnoid spaces enlargement. Moreover, the last MRI demonstrated normal cerebral liquoral spaces (Figure $3: \mathrm{d}, \mathrm{h}$ ) and the reduction of the size of parenchymal brain lesions (Figure 3: e-g). Spinal MRI showed post-traumatic severe spinal cord injury at the C1-C2 level with marked thinning and hyper-intensity of spinal cord damaged tissue; at this level Diffusion Tensor Tractography (DTT) detected severe spinal fibre loss (Figure 4, a-b).

\section{EEG studies}

EEG examinations performed before and after intranasal NGF administration showed a constant and progressive reduction in slow-wave activity with the recovery of a normal electrophysiological rhythm (figure not shown).

\section{Visual evoked potential studies (for protocol used, see Supplementary materials)}

Full-field $8 \mathrm{~Hz}$ Flicker visual evoked potentials (VEPs) were recorded at baseline and at the end of treatment according to a previously published technique (27). Compared with baseline, flicker VEP of both eyes showed a significant phase/ latency advance ( $>$ 90th percentile) of test-retest variability 


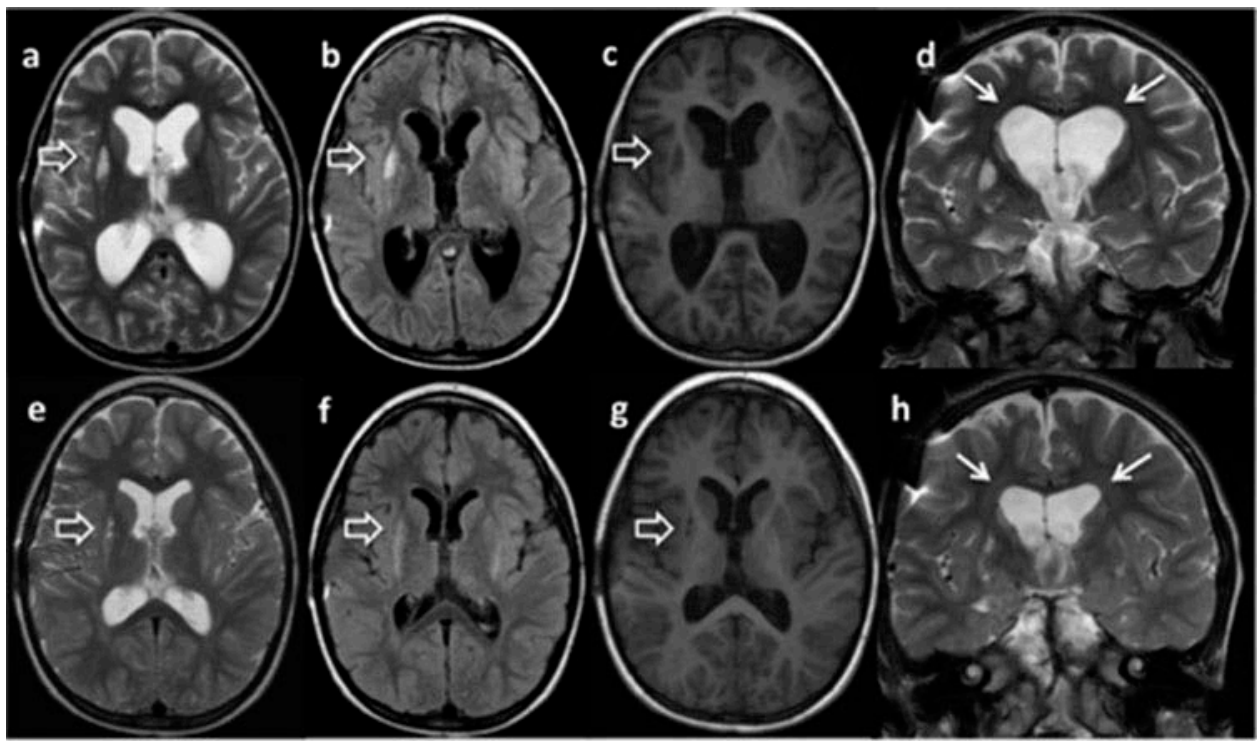

Figure 3. Brain MRI.

Brain MRI before (a-d) and after treatment with NGF (e-h). Axial T2w (a, e), FLAIR (b), T1w (c, g) axial images, coronal T2w (d, h) images. MRI before NGF treatment showed areas of abnormal T2w and FLAIR hyper-intensity ( $a$, b open arrows) and T1w hypo-intensity in the basal ganglia (c, open arrows), ventriculomegaly (d, white arrows) and subarachnoid spaces enlargement. At the end of NGF administration, brain MRI showed a marked reduction in ventricular dilatation and normal subarachnoid spaces ( $h$, white arrows); parenchymal lesions appeared small in size (e, f, g, open arrows).
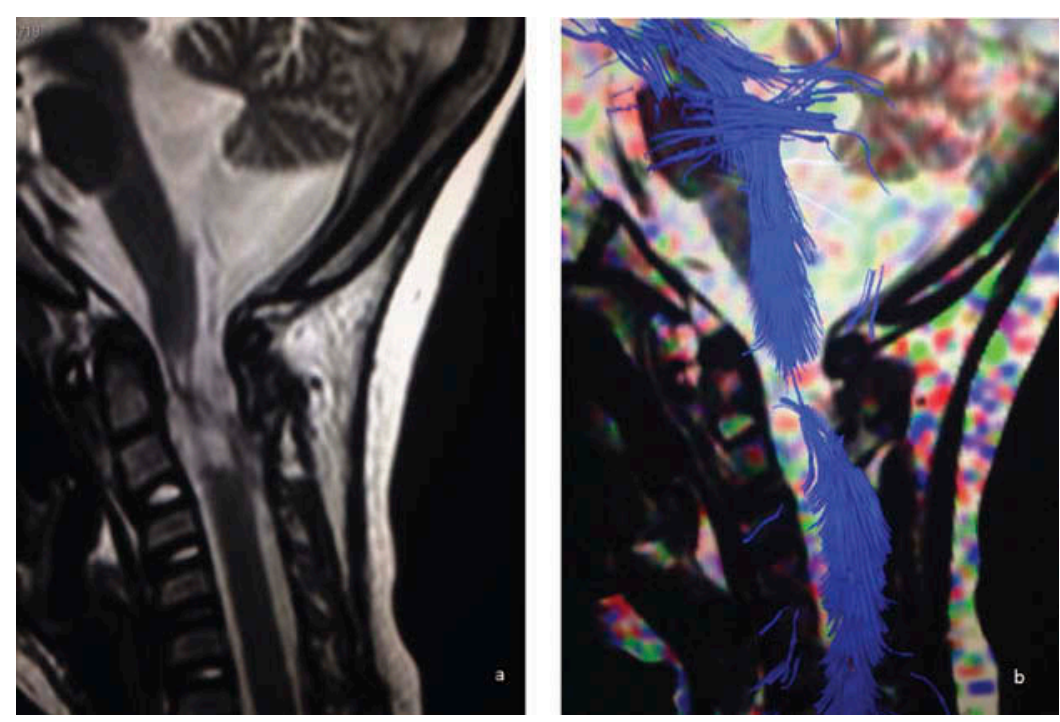

Figure 4. Cervical spine MRI.

Sagittal T2-weighted image (a), and Diffusion Tensor Tractography (DTT) (b) showing almost complete post-traumatic severe spinal cord injury at the C1-C2 level with marked thinning and hyper-intensity of damaged tissue; at this level DTT also showed severe fibre loss.

with a shift of 60 and 100 degrees for the right and left eyes, respectively. This shift corresponded to a response latency advance between 20 and 30 milliseconds, indicating faster and more efficient visual cortical responses to light after NGF treatment. Conversely, SSEP did not show any modifications after NGF treatment (figures not shown).

\section{NGF and DCX assay in the cerebrospinal fluid}

To assess whether intranasal NGF administration modifies the levels of soluble NGF detectable in the cerebrospinal fluid (CSF), NGF ELISA was performed on CSF samples collected by lumbar puncture, performed in deep sedation of the patient, before (pretreatment) and at the end of NGF administration (see Supplementary Materials for details on treatment protocol). As shown in Figure 5A, the NGF levels increased in the post-treatment CSF samples (Figure $5 \mathrm{~A}$ ). Indeed, there was a $+30 \%$ and $+44 \%$ of the NGF content in the CSF after the first and the fourth treatment, respectively. Notably, the basal NGF levels (pretreatment values) also increased (+15\%) before the last treatment was started. It has been previously reported that the CSF levels of the neurogenesis-associated protein DCX increase after intraventricular NGF infusion (26) and correlate with improved outcome in children with severe 

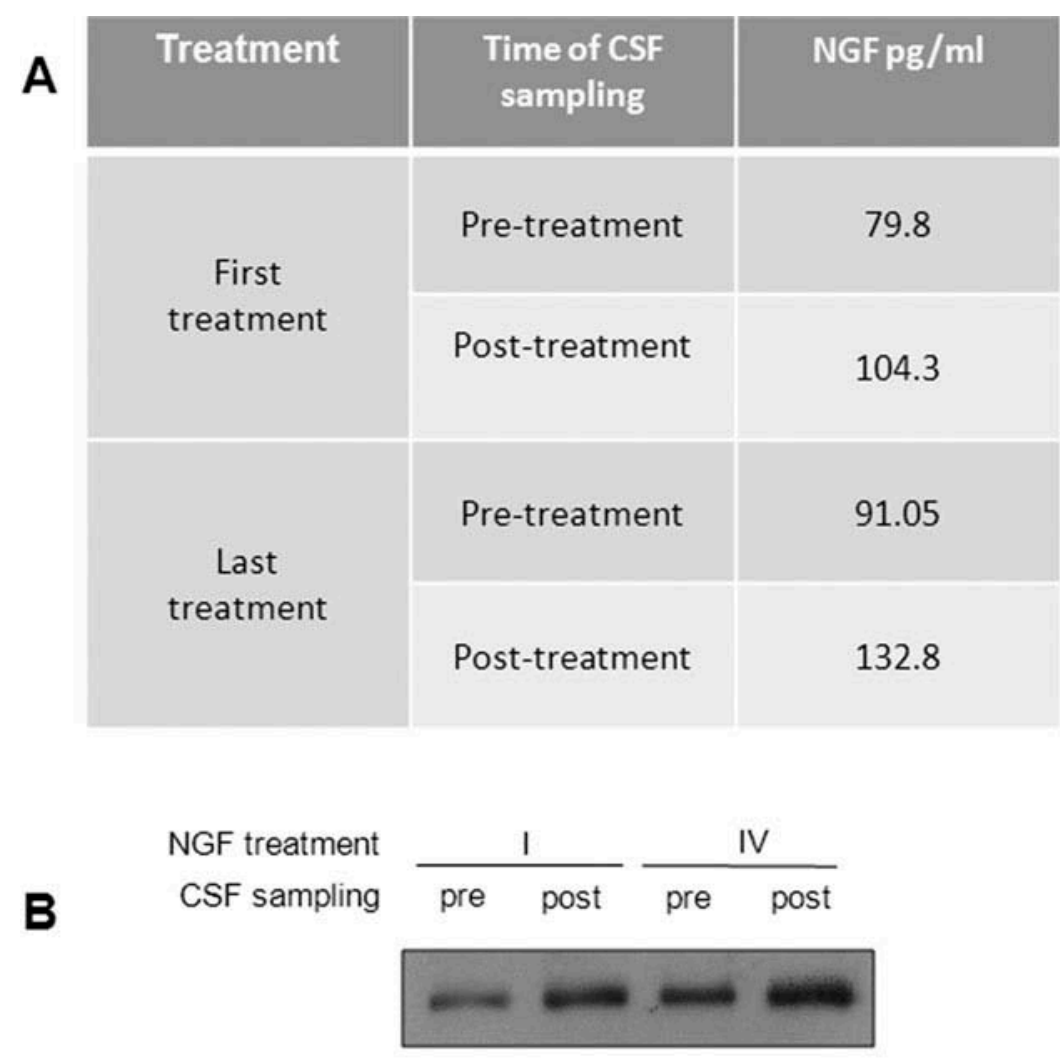

blot: anti - DCX

Figure 5. NGF and Doublecortin in the CSF.

The CSF content of NGF and DCX were analysed in the CSF samples collected before (pre) and after (post) the first and the last treatment period. (A) NGF content in the CSF, measured by NGF ELISA, increased by $30 \%$ and $44 \%$ after the first and the last intranasal NGF treatment run, respectively. A $15 \%$ increase was also observed when pretreatment samples were compared. Data expressed as $\mathrm{pg} / \mathrm{mL}$. Assays were performed in duplicate. (B) Western blot analysis of the same samples revealed that the CSF content of DCX increased after the intranasal NGF treatments. Moreover, the comparison of the two pretreatment samples also demonstrated a sustained basal increase of DCX induced by the first NGF treatment.

TBI (28). Then, in this case study Western blot has been used to detect the presence and variation of DCX levels in the CSF before and after intranasal NGF administration. As depicted in Figure 5B, DCX increased in the CSF in both the posttreatment samples, when compared with the respective pretreatment controls (Figure 5B). Moreover, similar to what was observed for NGF protein, the basal CSF content of DCX also increased with time, as evidenced by the comparison between the pretreatment samples.

\section{Discussion}

This case study reports about a four-year-old boy suffering from severe TBI, tetraplegia, neurological bladder and severe communicative and neuropsychological impairment due to the effects of primary brain injury and prolonged cardiopulmonary arrest. The devastating neurologic sequelae caused by cardiac arrest and cerebral hypoperfusion have been recognized since the early development of modern resuscitation techniques (29). The persistence of unfavourable neurologic outcome, despite advances in cardio-pulmonary resuscitation, led the American Heart Association to recognize brain injury after cardiac arrest as an important area for clinical research. In the last guidelines, the term 'cardiopulmonary-cerebral resuscitation' was proposed to emphasize brain injury in relation to cardiac arrest (30). Global cerebral ischaemia during cardiac arrest results in heterogeneous injury to the brain. Large projection neurons of the cerebral cortex, cerebellar Purkinje cells and the CA-1 area of the hippocampus are the most vulnerable sites (31), whereas the subcortical areas, such as the brainstem, thalamus and hypothalamus, are more resistant to injury (32). The landmark controlled clinical trials that primarily targeted brain injury after cardiac arrest span from the Brain Resuscitation Clinical Trial of barbiturates in 1986 (33) to the more recent trials of therapeutic hypothermia $(29,34)$; however, no conclusive results have been reported in improving the neurologic outcome in this kind of patients. Despite advanced and up-to-date critical, neurointensive and rehabilitative care, our patient showed no neurological improvement 6 months after brain injury, so the child was started on an experimental treatment with intranasal NGF administration. Intranasal NGF treatment was followed by a significant improvement of functional (PET/CT, SPECT/CT and MRI) and electrophysiological (EEG and VEP) results, with also a concomitant amelioration of the patient's clinical and neurological conditions, characterized by the acquisition of voluntary movements of his legs, arms and fingers, improved facial mimicry and phonation, attention and verbal comprehension with consequent better interaction with his parents and caregivers. After NGF treatment, the child 
recovered the ability to cry, cough reflex, control of oral motility and feeding capacity, showing an improvement also in bowel and urinary functions and, finally, making some spontaneous respiratory efforts. It is now accepted that TBI is the leading cause of a cascade of regenerative events that can last for several weeks. It is possible that part of the improvement in neurological conditions of the child could be explained by the physiologic spontaneous recovery after TBI. Many adaptive processes may begin early in the post-injury stage and result in neuroplastic changes leading to at the restoration of some neurological functions (35). A key role in post-TBI recovery process seems to be related to the postinjury oligodendrogenesis (36). Oligodendrocytes perform many functions in the normal brain, the best known of which is myelin production around axons; therefore, their role is considered fundamental in post-TBI neuroplasticity and circuitry function $(37,38)$. This crosstalk between axons and oligodendrocytes modulates the speed of signal conduction in an activity-dependent manner and highlights the role that oligodendrocytes play in enhancing plasticity (39). Therefore, it is possible that the neurologic improvement in our patient could in part depend on this spontaneous recovery, but it would have been too complicated to demonstrate how much of this. Neuroradiological, electrophysiological and clinical changes observed in our patient have been so remarkable and occurred in a relatively short time that probably they are related to NGF treatment rather than to a 'spontaneous recovery'. Moreover, behavioural experience has been recently recognized as the most potent modulator of brain plasticity after head injury, but it is possible to hypothesize that this type of stimuli was low compared with the effects of NGF administration (40). The extensive role of the neuroprotective mechanisms of NGF, in both central and peripheral nervous systems, has already been widely reported, mainly as part of neuronal damage caused by severe TBI $(26,41,42)$. Delivering neurotrophic factors into the brain has classically been a significant challenge owing to the presence of the blood-brain and blood-cerebrospinal fluid barriers limiting drug penetration into the CNS $(16,43)$. Accumulated experience has pointed to the existence of a direct pathway from the nose to the brain that can facilitate the release of drugs into the CNS. Although the exact mechanisms are not completely understood yet, growing evidences suggest that these pathways involve olfactory nerve terminations, trigeminal nerve fibres, vascular and lymphatic pathways that allow NGF to be actively delivered into the CNS $(44,45)$. In experimental animal models, intranasal NGF administration showed different actions after severe TBI, such as attenuating amyloid $\beta 42$ (A $\beta 42)$ deposits and brain oedema, inhibiting the transcription and expression of pro-inflammatory cytokines, minimizing mitochondria-mediated apoptosis and, finally, reducing TBIinduced elevation of interleukin $1 \beta$ (IL-1 $1 \beta$ ), thus significantly improving the neurological outcome of the injured brain $(19,46)$. This patient is the first child with severe TBI to be treated with intranasal NGF. This administration pathway afforded higher and more persistent concentrations of NGF in the CSF, suggesting that NGF applied on the intranasal mucosa can readily reach the CNS, as well as the cerebral cortex. Intranasal NGF improved cerebral perfusion and metabolism with the consequence of concomitant amelioration of neurological functions of the child, confirming that this neurotrophin exerts a key role in neuroprotective mechanisms in injured brain. The specific role of NGF in determining the mechanisms of neuronal repair in the damaged brain is not understood yet, but some evidences support the action of this neurotrophin in modulating DCX biosynthesis inside the brain. New striatum neurons can be generated when NGF is infused into the lateral ventricle of the brain and increased levels of intrastriatal neurotrophin promote the initial phase of induced neurogenesis (42). Previously, increased levels of both NGF and DCX have been showed to correlate significantly with a good outcome of patients with severe TBI (26). The correlation between NGF and DCX suggests that both these biological markers are involved in the mechanism of neuronal repair after TBI, thus playing a pivotal role in neuronal connection reorganization after brain insult. DCX-positive cells were present near and among the glial scars early after brain damage, migrating from the sub-ventricular zone towards the cerebral cortex lesions, thus facilitating the recovery of some cerebral functions in brain-injured patients (47). The impressive results obtained in our patient, using intranasal NGF administration, open a new potential therapeutic window not only offering rescue treatment in children suffering from severe TBI but also in the case of those ischaemic and neurodegenerative diseases responsible for the loss of neuronal function (48). The neuroprotective effects obtained by intranasal NGF are of particular importance considering the ease of neurotrophin application via the intranasal route and the possibility of repeating the drug administration without local or systemic side effects.

In conclusion, this is a first step towards the development of a larger clinical project aimed at evaluating the potential effectiveness of intranasal NGF administration for improving neurological outcome and clinical functions in children with severe TBI. The current preliminary findings and the ease of administration of the drug make it worthwhile to be investigated further, mainly in head-injury patients with better baseline neurological conditions, in order to explore more thoroughly the benefits of NGF on cerebral function recovery. Although further controlled, randomized, double-blind studies are needed for a better understanding of the neuroprotective mechanisms of this neurotrophin, intranasal NGF administration appears to be a promising and safe rescuing strategy for the treatment of children with neurological sequelae due to severe head injury. This is the first report of a nonaggressive treatment strategy with beneficial effects on neurological functions in a child with severe TBI.

\section{Declaration of interest}

The authors declare that they have no conflicts of interest concerning this article.

\section{References}

1. Centers for Disease Control and Prevention [Internet]. Report to congress. Traumatic Brain Injury in the United States: Epidemiology and Rehabilitation. Atlanta (GA); 2010. https:// 
www.cdc.gov/traumaticbraininjury/pdf/tbi_report_to_congress_ epi_and_rehab-a.pdf.

2. Centers for Disease Control and Prevention [Internet]. Rates of TBI-related emergency department visits by age group - United States, 2001-2010. https://www.cdc.gov/traumaticbraininjury/ data/rates_ed_byage.html.

3. Moppett IK. Traumatic brain injury: assessment, resuscitation and early management. Br J Anaesth. 2007;99:18-31.

4. Chiaretti A, Genovese O, Riccardi R, Riccardi R, Di Rocco C, Di Giuda D, Mariotti P, Pulitanò S, Piastra M, Polidori G, et al. Intraventricular nerve growth factor infusion: a possible treatment for neurological deficits following hypoxic-ischemic brain injury in infants. Neurol Res. 2005;27:741-46.

5. Manni L, Rocco ML, Bianchi P, Soligo M, Guaragna M, Barbaro SP, Aloe L. Nerve growth factor: basic studies and possible therapeutic applications. Growth Factors. 2013;31:115-22.

6. Mattson MP, Scheff SW. Endogenous neuroprotection factors and traumatic brain injury: mechanisms of action and implications for therapy. J Neurotrauma. 1994;1:3-33.

7. Garofalo L, Cuello AC. Nerve growth factor and the monosialoganglioside GM1: analogous and different in vivo effects on biochemical, morphological, and behavioral parameters of adult cortically lesioned rats. Exp Neurol. 1994;125:195-217.

8. Moser KV, Reindl M, Blasig I, Humpel C. Brain capillary endothelial cells proliferate in response to NGF, express NGF receptors and secrete NGF after inflammation. Brain Res. 2004;1017:53-60.

9. Dollé JP, Rezvan A, Allen FD, Lazarovici P, Lelkes PI. Nerve growth factor-induced migration of endothelial cells. J Pharmacol Exp Ther. 2005;315:1220-27.

10. Couillard-Despres S, Winner B, Schaubeck S, Aigner R, Vroemen M, Weidner N, Bogdahn U, Winkler J, Kuhn HG, Aigner L. Doublecortin expression levels in adult brain reflect neurogenesis. Eur J Neurosci. 2005;21:1-14.

11. Horesh D, Sapir T, Francis F, Wolf SG, Caspi M, Elbaum M, Chelly J, Reiner O. Doublecortin, a stabilizer of microtubules. Hum Mol Genet. 1999;8:1599-610.

12. Zigova T, Pencea V, Wiegand SJ, Luskin MB. Intraventricular administration of BDNF increases the number of newly generated neurons in the adult olfactory bulb. Mol Cell Neurosci. 1998;11:234-45.

13. Pan W, Banks WA, Kastin AJ. Permeability of the blood-brain barrier to neurotrophins. Brain Res. 1998;788:87-94.

14. Clinical Trials.gov [Internet]. NCT01212679. Effects of Intranasal Nerve Growth Factor for Traumatic Brain Injury. Jinling Hospital, China; 2010. https://clinicaltrials.gov/ct2/show/study/ NCT01212679.

15. Frey IW, Liu J, Chen X, Thorne RG, Fawcett JR, Ala TA, Rahman YE. Delivery of $125 \mathrm{I}-\mathrm{NGF}$ to the brain via the olfactory route. Drug Deliv. 1997;4:87-92.

16. Tian L, Guo R, Yue X, Lv Q, Ye X, Wang Z, Chen Z, Wu B, Xu G, Liu $X$. Intranasal administration of nerve growth factor ameliorate $\beta$-amyloid deposition after traumatic brain injury in rats. Brain Res. 2012;1440:47-55.

17. Young J, Pionk T, Hiatt I, Geeck K, Smith JS. Environmental enrichment aides in functional recovery following unilateral controlled cortical impact of the forelimb sensorimotor area however intranasal administration of nerve growth factor does not. Brain Res Bull. 2015;115:17-22.

18. De Rosa R, Garcia A, Braschi C, Capsoni S, Maffei L, Berardi N, Cattaneo A. Intranasal administration of NGF rescues recognition memory deficits in anti-NGF transgenic mice. Proc Natl Acad Sci USA. 2005;102:3811-16.

19. Lv Q, Fan X, Xu G, Liu Q, Tian L, Cai X, Sun W, Wang X, Cai Q, Bao $Y$, et al. Intranasal delivery of nerve growth factor attenuates aquaporins-4-induced edema following traumatic brain injury in rats. Brain Res. 2013;1493:80-89.

20. Seel RT, Sherer M, Whyte J, Katz DI, Giacino JT, Rosenbaum AM, Hammond FM, Kalmar K, Pape TL, Zafonte R, et al. American congress of rehabilitation medicine, brain injury-interdisciplinary special interest group, disorders of consciousness task force.
Assessment scales for disorders of consciousness: evidence-based recommendations for clinical practice and research. Arch Phys Med Rehabil. 2010;91:1795-813.

21. Laureys S, Celesia GG, Cohadon F, Lavrijsen J, León-Carrión J, Sannita WG, Sazbon L, Schmutzhard E, Von Wild KR, Zeman A, et al. European task force on disorders of consciousness. Unresponsive Wakefulness Syndrome: a new name for the vegetative state or apallic syndrome. European task force on disorders of consciousness. BMC Med. 2010;8:68.

22. Giacino JT, Ashwal S, Childs N, Cranford R, Jennett B, Katz DI, Kelly JP, Rosenberg JH, Whyte J, Zafonte RD, et al. The minimally conscious state: definition and diagnostic criteria. Neurology. 2002;58:349-53.

23. Middendorp JJ, Goss B, Urquhart S, Atresh S, Williams RP, Schuetz M. Diagnosis and prognosis of traumatic spinal cord injury. Global Spine J. 2011;1:1-8.

24. Catz A, Itzkovich M, Tesio L, Biering-Sorensen F, Weeks C, Laramee MT, Craven BC, Tonack M, Hitzig SL, Glaser E, et al. A multicenter international study on the Spinal Cord Independence Measure, version III: rasch psychometric validation. Spinal Cord. 2007;45:275-91.

25. Eriksdotter Jönhagen M, Nordberg A, Amberla K, Bäckman L, Ebendal T, Meyerson B, Olson L, Seiger SM, Theodorsson E, Viitanen $M$, et al. Intracerebroventricular infusion of nerve growth factor in three patients with Alzheimer's disease. Dement Geriatr Cogn Disord. 1998;9:246-57.

26. Chiaretti A, Antonelli A, Genovese O, Pezzotti P, Rocco CD, Viola L, Riccardi R. Nerve growth factor and doublecortin expression correlates with improved outcome in children with severe traumatic brain injury. J Trauma. 2008;65:80-85.

27. Falsini B, Ziccardi L, Lazzareschi I, Ruggiero A, Placentino L, Dickmann A, Liotti L, Piccardi M, Balestrazzi E, Colosimo C, et al. Longitudinal assessment of childhood optic gliomas: relationship between flicker visual evoked potentials and magnetic resonance imaging findings. J Neurooncology. 2008;88:87-96.

28. Chiaretti A, Antonelli A, Mastrangelo A, Pezzotti P, Tortorolo L, Tosi F, Genovese O. Interleukin-6 and nerve growth factor upregulation correlates with improved outcome in children with severe traumatic brain injury. J Neurotrauma. 2008;25:225-34.

29. Geocadin GR, Koenig MA, Jia X, Jia X, Stevens RD, Peberdy MA. Management of brain injury after resuscitation from cardiac arrest. Neurol Clin. 2008;26:487-506.

30. Guidelines ECG. Part I: introduction to the International Guidelines 2000 for CPR and ECC: a consensus on science. Circulation. 2000;102:1-179.

31. Wijdicks EF, Campeau NG, Miller GM. MR imaging in comatose survivors of cardiac resuscitation. Am J Neuroradiology. 2001;22:1561-65.

32. Fujioka M, Okuchi K, Sakaki T, Hiramatsu K, Miyamoto S, Iwasaki S. Specific changes in human brain following reperfusion after cardiac arrest. Stroke. 1994;25:2091-95.

33. Brain Resuscitation Clinical Trial I Study Group. Randomized clinical study of thiopental loading in comatose survivors of cardiac arrest. New England J Med. 1986;314:397-403.

34. Hypothermia after Cardiac Arrest Study Group. Mild therapeutic hypothermia to improve the neurologic outcome after cardiac arrest. New England J Med. 2002;346:549-56.

35. Cramer SC. Stroke recovery: how the computer reprograms itself. Neuronalplasticity: the key to stroke recovery. Kananskis, Alberta, Canada, 19-22 March 2000. Mol Med Today. 2000;6:301-03.

36. Takase H, Washida K, Hayakawa K, Arai K, Wang X, Lo EH, Lok $\mathrm{J}$. Oligodendrogenesis after traumatic brain injury. Behav Brain Res. 2016 Nov 6. doi:10.1016/j.bbr.2016.10.042.

37. Greer JE, McGinn MJ, Povlishock JT. Diffuse traumatic axonal injury in the mouse induces atrophy, c-Jun activation, and axonal outgrowth in the axotomized neuronal population. J Neurosci. 2011;31:5089-105.

38. Povlishock JT, Becker DP, Cheng CL, Vaughan GW. Axonal change in minor head injury. J Neuropathol Exp Neurol. 1983;42:225-42. 
39. Wake H, Ortiz FC, Woo DH, Lee PR, Angulo MC, Fields RD. Nonsynaptic junctions on myelinating glia promote preferential myelination of electrically active axons. Nat Commun. 2015;6:7844.

40. Nudo RJ. Recovery after brain injury: mechanisms and principles. Front Hum Neurosci. 2013;7:887.

41. Chiaretti A, Piastra M, Polidori G, Di Rocco C, Caresta E, Antonelli A, Amendola T, Aloe L. Correlation between neurotrophic factor expression and outcome of children with severe traumatic brain injury. Intensive Care Med. 2003;29:1329-38.

42. Chiaretti A, Antonelli A, Genovese O, Fernandez E, Giuda D, Mariotti P, Riccardi R. Intraventricular nerve growth factor infusion improves cerebral blood flow and stimulates doublecortin expression in two infants with hypoxic-ischemic brain injury. Neurol Res. 2008c;30:223-28.

43. Pradier P, Jalenques I, Dalle M, Reuling R, Despres G, Romand R. Distribution and metabolism patterns of plasma 7S- and betaNGF in the adult male rat. J Physiol Paris. 1994;88:273-77.
44. Dhuria SV, Hanson LR, Frey WH II. Intranasal delivery to the central nervous system: mechanisms and experimental considerations. J Pharm Sci. 2010;99:1654-73.

45. Hanson LR, Fine JM, Svitak AL, Faltesek KA. Intranasal administration of CNS therapeutics to awake mice. J Visualized Exp. 2013;8:212-17.

46. Lv Q, Lan W, Sun W, Ye R, Fan X, Ma M, Yin Q, Jiang Y, Xu G, Dai J, et al. Intranasal nerve growth factor attenuates tau phosphorylation in brain after traumatic brain injury in rats. J Neurol Sci. 2014;15:48-55.

47. Sundholm-Peters NL, Yang HK, Goings GE, Walker AS, Szele FG. Subventricular zone neuroblasts emigrate toward cortical lesions. J Neuropathol Exp Neurol. 2005;64:1089-100.

48. Covaceuszach S, Capsoni S, Ugolini G, Spirito F, Vignone D, Cattaneo A. Development of a non-invasive NGF-based therapy for Alzheimer's disease. Curr Alzheimer Res. 2009;6:15870. 